\title{
Differential Diagnosis of Acromegaly: Pachydermoperiostosis Two New Cases from Turkey
}

\author{
(D) Emine Kartal Baykan1, (D) Ayberk Türkyılmaz² \\ 1 Erzurum Regional Training and Research Hospital, Clinic of Endocrinology and Metabolic Diseases, Erzurum, Turkey \\ 2Karadeniz Technical University Faculty of Medicine, Department of Medical Genetics, Trabzon, Turkey
}

\begin{abstract}
What is already known on this topic?
Pachydermoperiostosis (PDP) is a rare condition characterised by digital clubbing, joint problems and pachydermia, but other skin manifestations due to dermal and sebaceous gland hypertrophy can be found.
\end{abstract}

\section{What this study adds?}

PDP cases mimicking acromegaly are reported to bring attention to this differential diagnosis. Although both acromegaly and PDP are infrequently encountered, avoidance of diagnostic confusion is important because of the prognostic and therapeutic implications. Awareness of the significance of clubbing under these circumstances is likely to prevent misdiagnosis.

\begin{abstract}
Pachydermoperiostosis (PDP), also known as primary hypertrophic osteoarthropathy, is a rare genetic disorder characterized by pachyderma and periostosis. Acromegaly is a condition caused by excessive secretion of growth hormone (GH) leading to elevated insulin-like growth factor 1 levels, and is characterised by somatic overgrowth and physical disfigurement, notably affecting hands and feet. We present two cases referred with an initial diagnosis of acromegaly that were ultimately diagnosed as PDP. Case 1: A 17 year-old boy presented with enlargement in both feet and hands, finger clubbing, swelling in knee joints, knee pain, coarsening of facial skin lines and forehead skin, and excessive sweating which increased gradually over five years. There were prominent skin folds on the forehead, face, and eyelids. Also, there was an enlargement in both hands and clubbing of the fingers. There was marked swelling in the knee joints and ankles. Genetic analysis revealed a novel homozygous variant NM_005630: c.31 C > T (p.Q11*) in the SLCO2A1 gene. Case 2: A 16 year-old boy presented with coarsening of forehead skin and scalp, excessive sweating, and pain in the elbow and knee over three years. Skin folds were prominent on the forehead and scalp. Genetic analysis revealed a homozygous variant NM_005630.2:c.86delG (p.G29Afs*48) in the SLCO2A1 gene. Such clinical presentation contemporaneous with normal GH level and prominent radiological abnormalities prompted the diagnosis of PDP. In conclusion, PDP is a very rare osteoarthrodermopathic disorder with clinical and radiographic presentation that may mimic acromegaly. In the evaluation of patients with acromegaloid appearance, PDP should be considered as a differential diagnosis.
\end{abstract}

Keywords: Acromegaly, pachydermoperiostosis, diagnosis

\section{Introduction}

Pachydermoperiostosis (PDP), also known as primary hypertrophic osteoarthropathy, is a rare genetic disorder characterized by pachyderma and periostosis. In 1935, the disorder was classified into three forms by three French dermatologists, Touraine, Solente, and Gole: complete (pachyderma and periostosis), incomplete (without pachyderma), and rough (pachyderma and minimal skeletal changes) forms (1). Diagnosis should be made when two of the following features are present: positive family history, hypertrophic skin changes, osseous pain/radiographic changes, or clubbing (2). Although the exact incidence is unknown in PDP, its prevalence is estimated at $0.16 \%$ (3). It generally manifests at puberty and the male:female ratio is $7: 1$ (4). The clinical features, signs and symptoms have 
close similarities with acromegaly and can cause diagnostic confusion (5). We present two cases referred with an initial diagnosis of acromegaly and were ultimately diagnosed as PDP.

\section{Case Reports}

\section{Case 1}

A 17 year-old boy initially presented to the family doctor with enlargement in both feet and hands and excessive sweating over five years, and he was referred to an endocrine outpatient clinic with an initial diagnosis of acromegaly. The patient's parents are cousins and the patient has a brother and a sister. There was no finding of similar complaints in their family history. The anamnesis revealed that his complaints had been worsening gradually over five years and that there was an enlargement in both feet and hands, clubbing in the fingers, swelling in knee joints, knee pain, coarsening of facial lines, particularly in the forehead skin, and excessive sweating. On physical examination, anthropometric measurements were: height, $183 \mathrm{~cm}$ [standard deviation score (SDS: 1.24)]; body weight, $79 \mathrm{~kg}$ (SDS: 0.89); and fathom distance, $183 \mathrm{~cm}$. There were prominent skin folds on the forehead, face, and eyelids
(Figure 1). There was also an enlargement in both hands and clubbing of the fingers (Figure 1). There was marked swelling in knee joints and ankles (Figure 1). Secondary sex characteristics were normal and the Tanner stage was five. Systemic examination, including cardiovascular and respiratory systems, neurological examination, and thyroid was normal. There was no scaling on the scalp, rash, psoriatic nail changes, subcutaneous nodules, or red-eye on physical examination. Complete blood count, hepatic and renal functions were within the normal range. Thyroid functions, follicle-stimulating hormone (FSH), luteinizing hormone (LH), total testosterone, prolactin, rheumatoid factor, and anti-cyclic citrullinated peptide were normal. Blood gas analysis showed: $\mathrm{pH}, 7.36$; $\mathrm{HCO}_{3}, 24.7$; and $\mathrm{SO}_{2}, 98 \%$. C-reactive protein was increased at $39.8 \mathrm{mg} / \mathrm{L}$ (reference: 0-5). Serological tests were performed to evaluate connective tissue disorders and vasculitis, which were all unremarkable. As acromegaly was suspected, insulin-like growth factor-1 (IGF-1) and growth hormone (GH) measurements were performed and the results were IGF-1 $235 \mathrm{ng} / \mathrm{mL}$ (normal: 111-509) and fasting $\mathrm{GH}$ $0.35 \mathrm{mcg} / \mathrm{L}$ (normal: 0.07-5). The oral glucose tolerance test (OGTT) was normal, suppression of GH less than $1 \mu \mathrm{g} / \mathrm{L}$. On bilateral hand radiographs, periostitis and hyperostosis were detected at the metacarpal and proximal phalanges
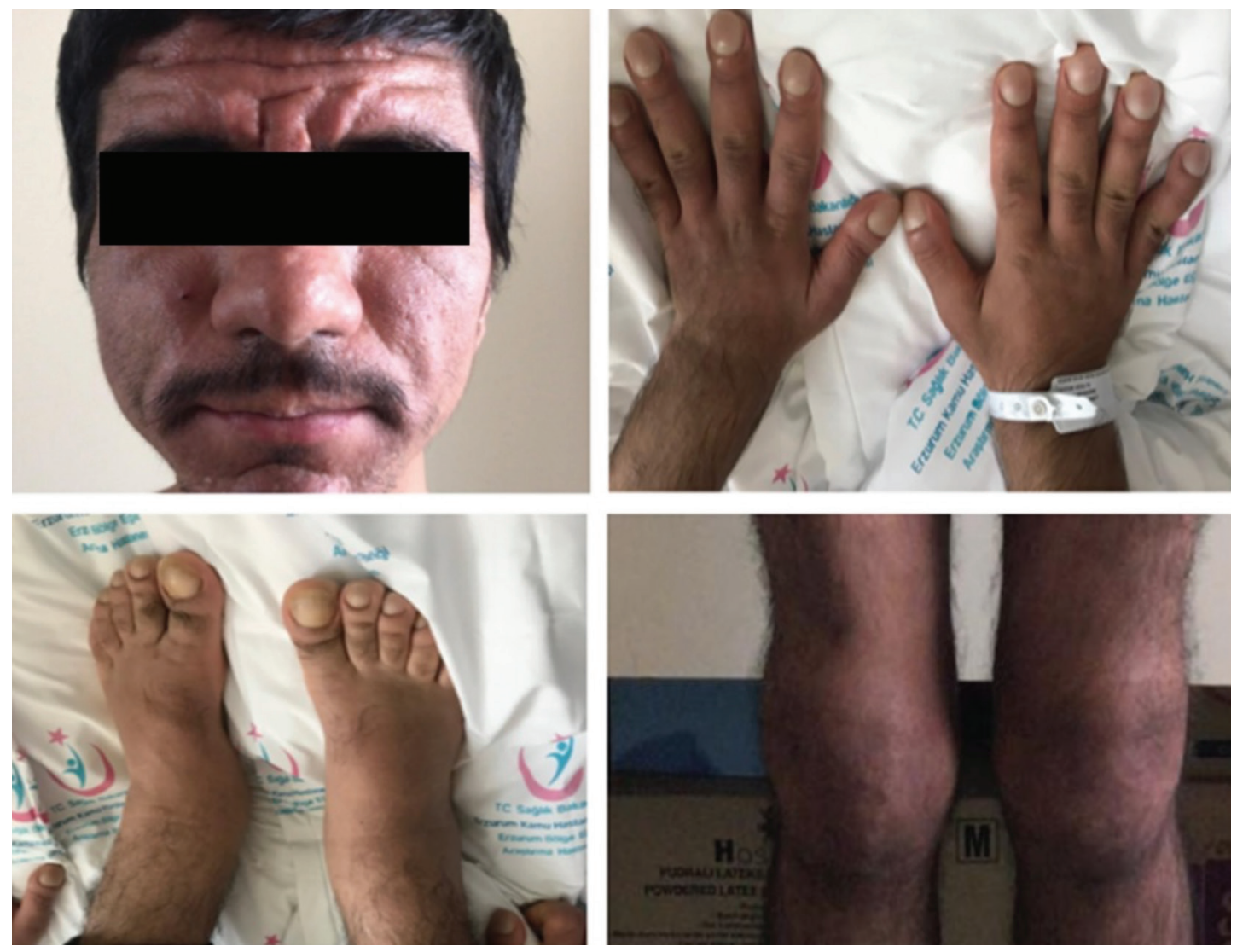

Figure 1. Physical examination findings of the first case 
(Figure 2). De novo bone formation and cortical thickening were detected on bilateral knee radiographs (Figure 2). Irregular sub-periosteal de novo bone formation and cortical thickening were observed in the tibia, fibula, calcaneum, and talus on bilateral ankle radiographs (Figure 3). No pituitary adenoma was detected on pituitary magnetic resonance imaging (MRI). Chest radiograph and echocardiography were considered normal. No abnormal finding was detected in abdominal and thoracic computed tomography (CT) scans. The patient was diagnosed as a classical PDP, based on clinical, biochemical, and radiological findings. Genetic analysis revealed a novel homozygous nonsense variant NM_005630:c.31 C > T (p.Q11*) in exon 1 of the solute carrier organic anion transporter family member 2A1 (SLCO2A1) gene. Thus, selective Cox-2 inhibitor (oral meloxicam, 15 mg twice daily) and steroid (oral methylprednisolone, 5 mg/ day) were prescribed. Marked improvement was detected in joint pain, swelling, and sweating in the first month after start of treatment. In the control visit at six months, regression was detected in the thickening at the forehead.

\section{Case 2}

An 16 year-old boy with coarsening of forehead skin and scalp, excessive sweating, and pain in the elbow and knee over three years was referred to an endocrine outpatient clinic with an initial diagnosis of acromegaly. There was no finding of a similar disorder in the family history, nor consanguinity between parents. The patient has a sister. On physical examination, anthropometric measurements were: height, $176 \mathrm{~cm}$ (SDS: 0.39); body weight, 69 kg (SDS: 0.27); and fathom distance, $176 \mathrm{~cm}$. Skin folds were prominent on the forehead skin and scalp (Figure 4). No abnormal finding was detected in the examination of the elbow and knee joint. Secondary sex characteristics were normal and the Tanner stage was five. Systemic examinations, including cardiovascular and respiratory systems, neurological examination, and thyroid, were normal. Complete blood count, hepatic and renal functions were within the normal range. Blood gas analysis showed: $\mathrm{pH}, 7.38$; $\mathrm{HCO}_{3}, 23.8$; and $\mathrm{SO}_{2}, 99 \%$. C-reactive protein was found to be increased at $11.4 \mathrm{mg} / \mathrm{L}$ (reference: 0-5). Thyroid functions, FSH, LH,

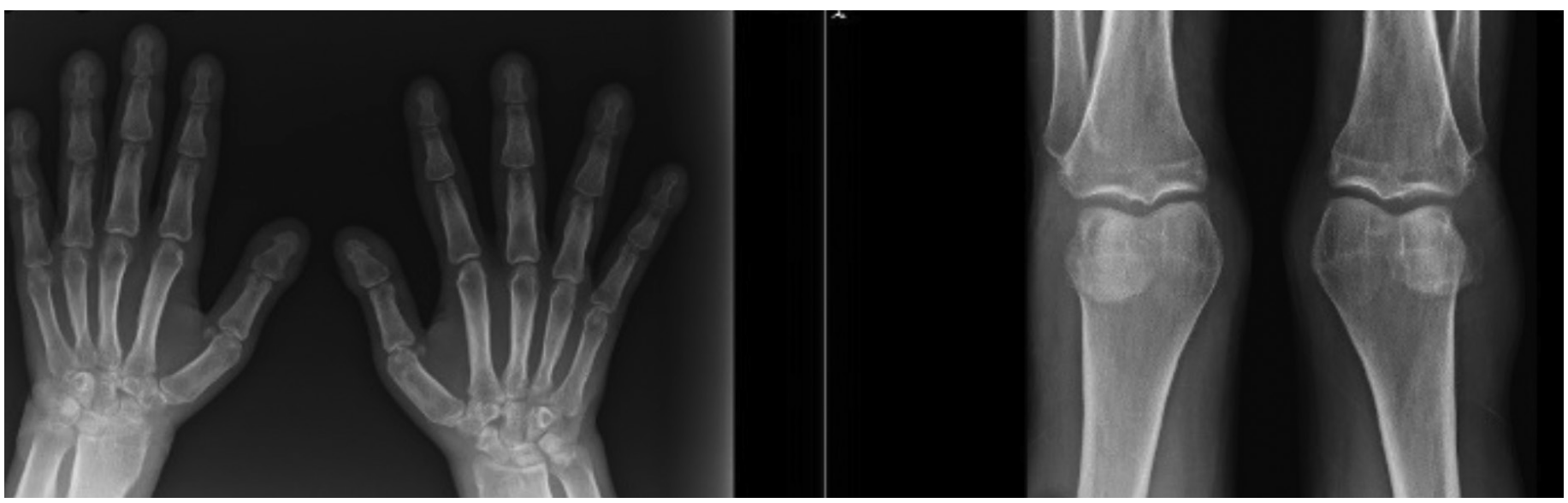

Figure 2. Radiography images of the first case

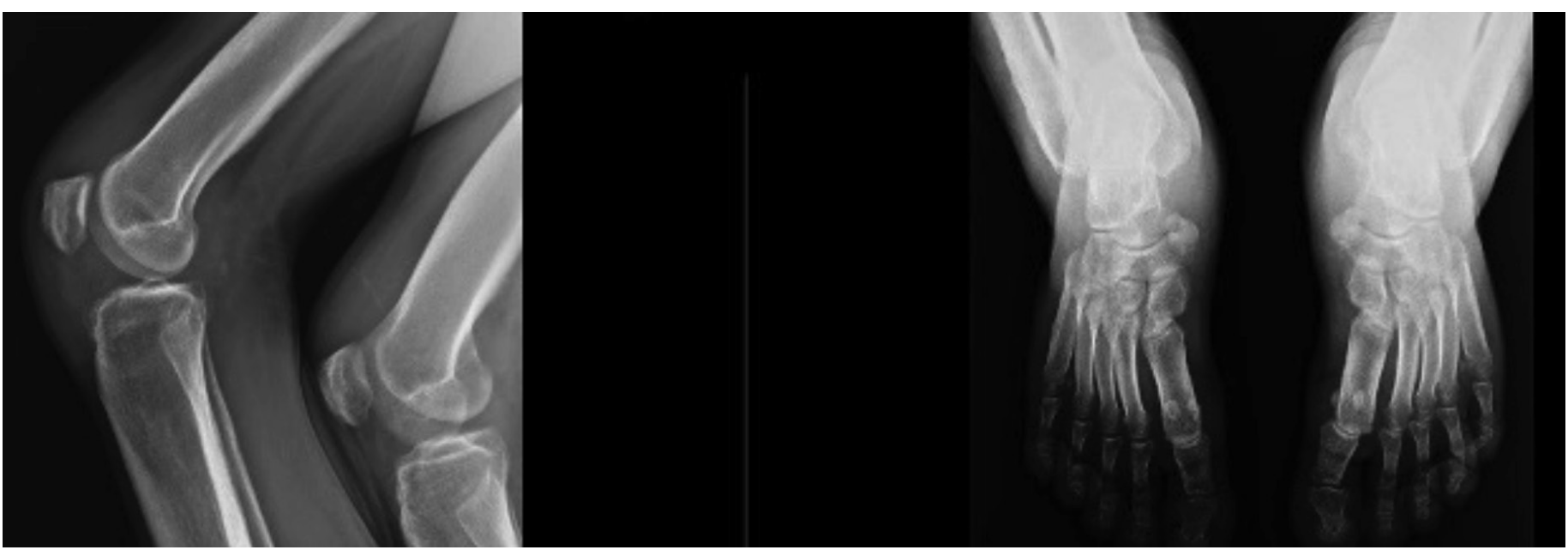

Figure 3. Radiography images of the first case 
total testosterone, prolactin, rheumatoid factor, and anticyclic citrullinated peptide were normal. Serological tests were performed to evaluate connective tissue disorder and vasculitis, which were again all normal. As acromegaly was suspected, IGF-1 and GH measurements were performed and the following values were obtained: IGF-1 $311 \mathrm{ng} / \mathrm{mL}$ (111-509) and fasting GH $0.45 \mathrm{mcg} / \mathrm{L}(0.07-5)$. The OGTT was normal, suppression of GH less than $1 \mu \mathrm{g} / \mathrm{L}$. Bilateral knee and hand radiographs were considered normal (Figure 5). No pituitary adenoma was detected on pituitary MRI. Chest radiograph and echocardiography were considered normal. No abnormal finding was detected in abdominal and

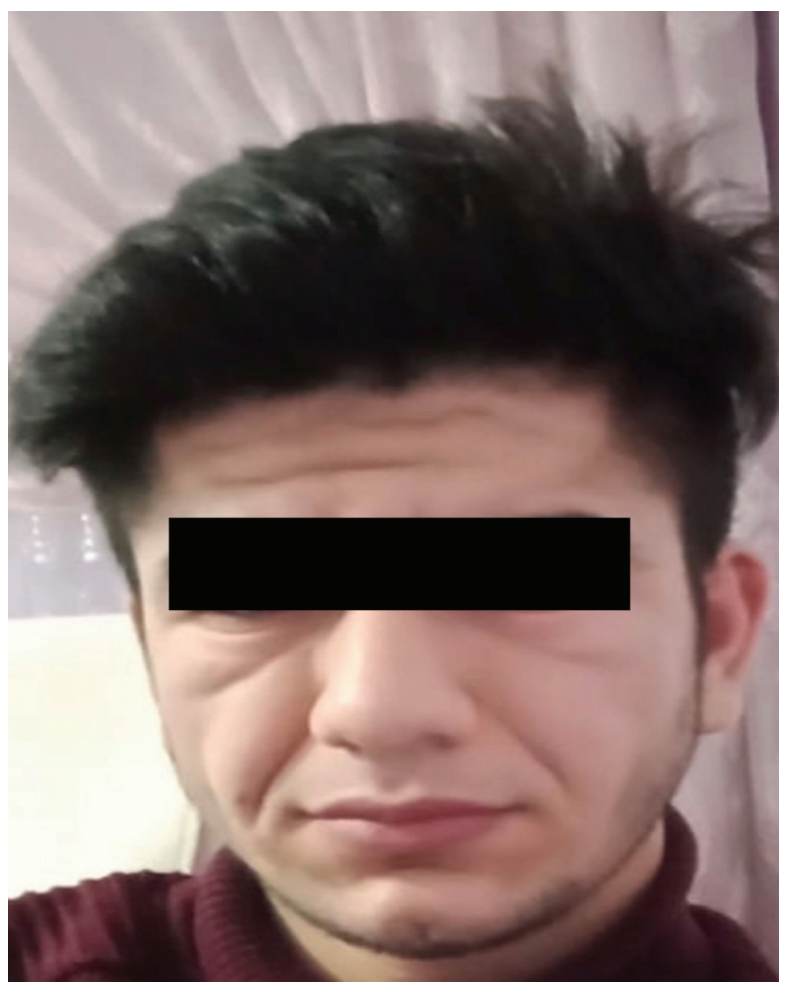

Figure 4. Physical examination findings of the second case thoracic CT scans. The patient was diagnosed with a rough form of PDP, based on clinical, biochemical, and radiological findings. Genetic analysis revealed a homozygous frameshift variant NM_005630.2:c.86delG (p.G29Afs*48) in exon 1 of the SLCO2A1 gene. This variant was previously reported in the ClinVar database as pathogenic. Thus, selective Cox-2 inhibitor (meloxicam, $15 \mathrm{mg}$ twice daily, PO) and steroid (methylprednisolone, $5 \mathrm{mg} /$ day, PO) were prescribed. On day six, melena developed in the patient; thus, he underwent esophagogastroduodenoscopy which revealed a duodenal ulcer $(2 \mathrm{~cm}$ in diameter). The meloxicam and prednisolone therapy were withdrawn and hydroxychloroquine was prescribed (200 mg twice daily, PO). After one month, regression was reported in joint pain and sweating. Marked regression was detected in skin thickening by month six.

\section{Discussion}

PDP is a rare hereditary disorder, for which the inheritance pattern has not yet been fully elucidated (6). The first case with PDP was reported by Friedreich in 1868. Touraine, Solente, and Gole defined PDP as a variant of hypertrophic osteoarthropathy, secondary to acromegaly and malignancy (1). As noted, PDP predominantly affects males and both patients reported here were boys (4). PDP begins during childhood or adolescence and progresses gradually over the next 5-20 years, before stabilizing (5). The typical presentations include thickening and coarsening of skin and/or scalp (pachydermia), clubbing of digits, oedema in the lower legs, arthritis both with and without joint effusion, and periostosis (swelling of periarticular tissue and subperiosteal new bone formation). The most common findings are associated with polyarthritis, cutis verticis gyrata, seborrhea and hyperhidrosis. PDP can manifest in three forms: classical or complete form (skin thickening, skeletal changes, and clubbing at fingers), incomplete form

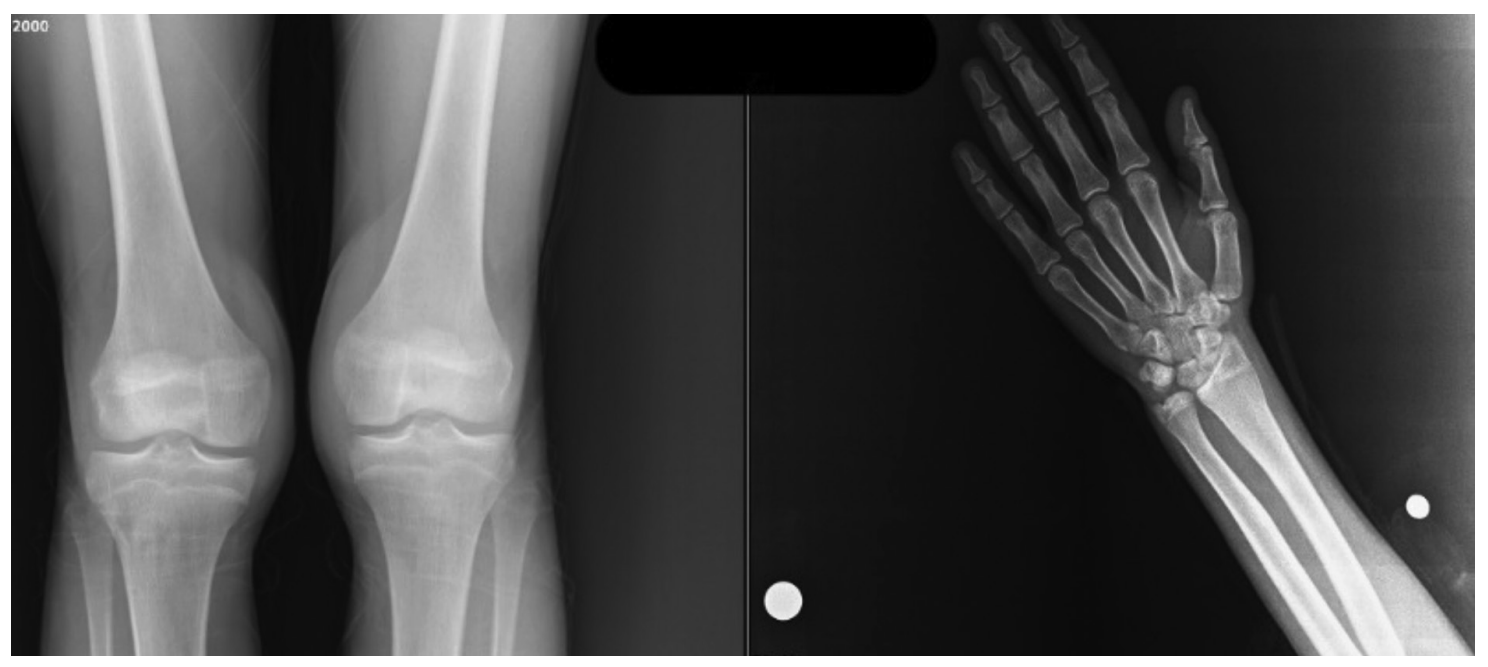

Figure 5. Radiography images of the second case 
(skeletal changes without skin involvement), and rough form (minimal skeletal change and skin thickening) (1).

PDP is very rare, and its clinical and radiological presentations can be confused with those of acromegaly, pseudoacromegaly with severe insulin resistance, secondary hypertrophic osteoarthropathy, Marfan's syndrome, McCune Albright syndrome, syphilitic periostitis, psoriatic onycho-pachydermo-periostitis and Paget's disease $(5,6,7,8,9,10,11)$. Acromegaly may bedue to sporadic or familial GH-secreting adenomas, arising during childhood or puberty. These features that are present in patients with acromegaly are due to the effect of excess GHs, mainly from pituitary tumours (12). The clinical picture in children and adolescents varies depending on whether the epiphyseal growth plate is open. Before epiphyseal fusion, there is a significant acceleration in growth rate, a condition also known as 'gigantism' but once epiphyseal fusion is complete, clinical symptoms become more similar to those in acromegalic adults, including coarse facial features, broadened nose, large hands and feet, organomegaly, and sweating (13). In acromegaly, excessive GH/IGF-1 production leads to periosteal bone formation, growth of synovial tissue, cartilage and precursor hypertrophic arthropathy associated with pain, and deformity, as seen in PDP. Acral abnormalities associated with PDP may overlap with those seen in acromegaly, including enlarged limbs, enlarged, thickened and short fingers, and thickened soft tissue. While digital clubbing and periostosis are seen in PDP, these findings are not seen in acromegaly (14). Facial coarsening, cutis verticis gyrate, seborrhea, acne and hyperhidrosis, are common in both PDP and in acromegaly. Symptoms specific to PDP but not seen in acromegaly, are long eyelashes, blepharoptosis, myelofibrosis, hypoalbuminemia, peptic ulcer, gastric cancer or watery diarrhoea in response to certain triggers, such as cold drinks, greasy food or sexual activity (15).

The pathogenesis of PDP is not yet clearly understood but evidence suggests that vascular endothelial growth factor (VEGF) and platelet-derived growth factor could play a central role (6). Recent studies demonstrated a prostaglandin mediated pathway as the key player in the pathogenesis of PDP. The 15-hydroxyprostaglandin dehydrogenase (HPGD) enzyme plays an important role in prostaglandin degradation and increased prostaglandin levels, particularly prostaglandin E2 (PGE2) (16). The two major genetic lesions both lead to an increase in PGE2, either by decreased degradation due to enzymatic loss (HPGD mutations) or a transporter defect (with SLCO2A1 mutations). The genetic assay was performed in both cases in this report, revealing a novel homozygous SLCO2A1 NM_005630.2: c.31 C>T $\left(\mathrm{p} . \mathrm{Q} 11^{*}\right)$ mutation in the first case and a previously reported homozygous SLCO2A1NM_005630.2:c.86delG (p.G29Afs*48) mutation in the second case. Radiological findings in PDP include subperiosteal newbone formation, cortical thickening, and narrowing of the joint spaces. Resorption of bone of the distal phalanges and ossification of inter-osseous membranes and ligaments can also be seen (17). In our first case, bilateral hand radiographs revealed periostitis and hyperostosis in the metacarpal and proximal phalanges. In our second case, radiographic examinations were found to be normal.

There is no specific treatment modality for PDP. Both 15 HPGD and SLCO2A1 genes are involved in PGE2 synthesis $(18,19)$. COX inhibitors (non-steroidal anti-inflammatory drugs, acetylsalicylic acid and corticosteroid) that inhibit the COX enzyme and suppress PGE2 biosynthesis are promising agents in PDP treatment. We achieved a good response in the first case, but treatment was discontinued in the second case due to upper gastrointestinal bleeding from a duodenal ulcer. In the first case, after six months of meloxicam and prednisolone treatment, the patient's complaints regressed significantly, so prenisolone treatment was discontinued by titration and followed up with meloxicam treatment. Alessandrella et al. (20) observed a marked improvement in skin findings and joint pain with hydroxychloroquine in a PDP patient with a homozygous SLCO2A1 gene mutation. In the second case in our report, hydroxychloroquine therapy was initiated as a homozygous SLCO2A1 gene mutation was detected, and marked regression was achieved in skin findings. Other agents used in medical therapy of PDP include aescin, bisphosphonate, colchicine, retinoids, tricyclic antidepressants, and tamoxifen citrate $(21,22)$. Botulinum toxin A has been used for cosmetic reasons. Surgery has been used to correct bone deformity, if present, and plastic surgery can be used to repair thickening in the forehead skin.

When a patient is suspected of having acromegaly, the first step is biochemical testing to confirm the clinical diagnosis, followed by imaging to determine the cause of excessive GH secretion. Pituitary adenoma is present in more than 95 percent of cases (23). The best single test for the diagnosis of acromegaly is measurement of serum IGF-1. Both serum GH concentrations and IGF-1 concentrations are increased in virtually all patients with acromegaly. During OGTT, serum GH level <1 $\mu \mathrm{g} / \mathrm{L}$ means the diagnosis of acromegaly is excluded. In PDP patients, IGF-1 and GH are normal and there is no adenoma in the pituitary (20). In both of our patients, GH and IGF-1 were found to be normal and $\mathrm{GH}$ after OGTT was $<1 \mathrm{ng} / \mathrm{mL}$ while pituitary MRI reported no adenoma. Although transsphenoidal surgery is recommended as the first step in treatment, treatment with 
a long-acting somatostatin analogue is also used in cases that do not respond to surgery (24).

\section{Conclusion}

In conclusion, the clinical presentation of PDP can be confused with multiple other diagnoses, especially acromegaly. Therefore PDP should be considered in the differential diagnosis of individuals presenting with acromegaloid feature.

\section{Ethics}

Ethics Committee Approval: All procedures performed during this retrospective study were following the ethical standards of the institutional and/or national research committee and with the 1964 Helsinki Declaration and its later amendments or comparable ethical standards. Ethical committee approval is not required for case reports.

Informed Consent: Informed consent forms were obtained from the patients and their families.

\section{Authorship Contributions}

Surgical and Medical Practices: Emine Kartal Baykan, Ayberk Türky1lmaz, Concept: Emine Kartal Baykan, Ayberk Türkyılmaz, Design: Emine Kartal Baykan, Ayberk Türkyılmaz, Data Collection or Processing: Emine Kartal Baykan, Ayberk Türkyılmaz, Analysis or Interpretation: Emine Kartal Baykan, Ayberk Türkyllmaz, Literature Search: Emine Kartal Baykan, Ayberk Türkyılmaz, Writing: Emine Kartal Baykan.

Financial Disclosure: The authors declared that this study received no financial support.

\section{References}

1. Thomas RH, Kirby JD. Pachydermoperiostosis with multiple basal cell carcinomata. J R Soc Med 1985;78:335-337.

2. Salah BI, Husari KI, Hassouneh A, Al-Ali Z, Rawashdeh B. Complete primary pachydermoperiostosis: A case report from Jordan and review of literature. Clin Case Rep 2019;7:346-352

3. Doshi D. Touraine-Solente-Gole syndrome. Orbit 2018;37:97-101 Epub 2017 Oct 17

4. Reginato AJ, Schiapachasse V, Guerrero R. Familial idiopathic hypertrophic osteoarthropathy and cranial suture defects in children. Skeletal Radiol 1982:8:105-109.

5. Abdullah NRA, Jason WLC, Nasruddin AB. Pachydermoperiostosis: a rare mimicker of acromegaly. Endocrinol Diabetes Metab Case Rep 2017;2017:17-0029.

6. Uppal S, Diggle CP, Carr IM, Fishwick CW, Ahmed M, Ibrahim GH, Helliwell PS, Latos-Bieleńska A, Phillips SE, Markham AF, Bennett CP,
Bonthron DT. Mutations in 15-hydroxyprostaglandin dehydrogenase cause primary hypertrophic osteoarthropathy. Nat Genet 2008;40:789793.

7. Berlin L. Duke Radiology Case Review: Imaging, Differential Diagnosis, and Discussion. JAMA 2012;307:2199-2200.

8. Boisseau-Garsaud AM, Beylot-Barry M, Doutre MS, Beylot C, Baran R. Psoriatic onycho-pachydermo-periostitis. A variant of psoriatic distal interphalangeal arthritis? Arch Dermatol 1996;132:176-180.

9. Auger DM, Stavrianeas N. Pachydermoperiostosis Member of The European Editorial Committee of Orphanet Encyclopedia, 2004.

10. Chakraborty RK, Sharma S. Secondary Hypertrophic Osteoarthropathy. In: StatPearls. Treasure Island (FL): StatPearls Publishing, 2020.

11. Ulutaş F, Ulu M, Karasu U, Çobankara V. Hypertrophic Osteoarthropathy Presenting as Rheumatoid Arthritis Mimicker: A Case Report. Cureus 2020;12:e9271

12. Pandey P, Ojha BK, Mahapatra AK. Pediatric pituitary adenoma: A series of 42 patients. J Clin Neurosci 2005;12:124-127.

13. Laws ER Jr, Scheithauer BW, Carpenter S, Randall RV, Abboud CF. The pathogenesis of acromegaly. Clinical and immunocytochemical analysis in 75 patients. J Neurosurg 1985;63:35-38.

14. Pineda CJ, Martinez-Lavin M, Goobar JE, Sartoris DJ, Clopton P, Resnick D. Periostitis in hypertrophic osteoarthropathy: relationship to disease duration. AJR Am J Roentgenol 1987;148:773-778.

15. Marques P, Stelmachowska-Banas M, Collier D, Wernig F, Korbonits M. Pachydermoperiostosis mimicking the acral abnormalities of acromegaly. Endocrine 2020;67:499-500. Epub 2020 Jan 8

16. Tanese K, Niizeki H, Seki A, Otsuka A, Kabashima K, Kosaki K, Kuwahara M, Miyakawa S, Miyasaka M, Matsuoka K, Okuyama T, Shiohama A, Sasaki T, Kudoh J, Amagai M, Ishiko A. Pathological characterization of pachydermia in pachydermoperiostosis. J Dermatol 2015;42:710-714.

17. Mangupli R, Daly AF, Cuauro E, Camperos P, Krivoy J, Beckers A. Primary hypertrophic osteoarthropathy due to a novel SLCO2A1 mutation masquerading as acromegaly. Endocrinol Diabetes Metab Case Rep 2017;2017:17-0013.

18. Nakanishi T, Nakamura Y, Umeno J. Recent advances in studies of SLCO2A1 as a key regulator of the delivery of prostaglandins to their sites of action. Pharmacol Ther 2021;223:107803. Epub 2021 Jan 16

19. Kozak KR, Milne GL, Morrow JD, Cuiffo BP. Hypertrophic osteoarthropathy pathogenesis: A case highlighting the potential role for cyclo-oxygenase-2-derived prostaglandin E2. Nat Clin Pract Rheumatol 2006;2:452-456.

20. Alessandrella A, Della Casa R, Alessio M, Puente Prieto J, Strisciuglio P, Melis D. A novel homozygous mutation in the SLCO2A1 gene causing pachydermoperiostosis: Efficacy of hydroxychloroquine treatment. Am J Med Genet A 2018;176:1253-1257.

21. 21 Zhang H, Yang B. Successful treatment of pachydermoperiostosis patients with etoricoxib, aescin, and arthroscopic synovectomy: Two case reports. Medicine (Baltimore) 2017;96:e8865

22. Chakraborty PP, Bhattacharjee R, Mukhopadhyay S, Chowdhury S. Pseudoacromegaly in pachydermoperiostosis. BMJ Case Rep 2016;2016:bcr2016214624.

23. Melmed S. Medical progress: Acromegaly. N Engl J Med 2006;355:25582573. Erratum in: N Engl J Med 2007;356:879.

24. Katznelson L, Laws ER Jr, Melmed S, Molitch ME, Murad MH, Utz A, Wass JA; Endocrine Society. Acromegaly: an endocrine society clinical practice guideline. J Clin Endocrinol Metab 2014;99:3933-3951. Epub 2014 Oct 30 